\title{
Perioperative Implications of Vascular Endothelial Dysfunction: Current Understanding of this Critical Sensor-Effector Organ
}

\author{
Bernhard Riedel $\cdot$ Neysan Rafat $\cdot$ Kim Browne • \\ Kate Burbury $\cdot$ Robert Schier
}

Published online: 22 June 2013

(C) Springer Science + Business Media New York 2013

\begin{abstract}
The endothelium, a delicate monolayer of cells lining all blood vessels, is a highly responsive sensoreffector organ, which through the secretion of a multitude of mediators, controls vascular tone, interacts with the inflammatory-coagulation cascades, promotes anti-aggregation, regulates immune cellular trafficking, and is crucial to angiogenesis. In brief, the endothelium is a major determinant of vascular homeostasis. It is easily damaged by intrinsic and extrinsic factors, and given its limited capacity for self-repair, is dependent on circulating bone marrow-derived endothelial progenitor cells for regeneration. There is increasing recognition that targeting the endothelium and maximizing inherent physiological function in the perioperative period may improve postoperative outcomes. This review explores the pathogenesis of endothelial dysfunction, the clinical utility of noninvasive methods to assess preoperative endothelial/microvascular (dys)function to improve risk stratification, and potential
\end{abstract}

B. Riedel $(\square) \cdot$ K. Browne

Department of Anesthesiology, Pain and Perioperative Medicine, Peter MacCallum Cancer Centre, University of Melbourne, Melbourne, VIC, Australia

e-mail: bernhard.riedel@petermac.org

N. Rafat

Department of Pediatrics I, University Children's Hospital Heidelberg, Heidelberg, Germany

\section{K. Burbury}

Department of Hematology, Peter MacCallum Cancer Centre, University of Melbourne, Melbourne, VIC, Australia

R. Schier

Department of Anaesthesiology and Intensive Care Medicine, University Hospital of Cologne, Cologne, Germany strategies for perioperative modulation of endothelial function.

Keywords Endothelium - Bone marrow $\cdot$ Endothelial progenitor cells (EPC) · Perioperative · Surgical · Postoperative $\cdot$ Outcomes · Flow mediated dilation - FMD · Statins $\cdot$ Exercise $\cdot$ Brachial artery reactivity testing

\section{Introduction}

It is well established that the endothelium, a delicate monolayer of cells lining all blood vessels, is a highly responsive sensor-effector organ, which through the secretion of a multitude of mediators controls vascular tone, interacts with the inflammatory-coagulation cascades, promotes anti-aggregation, regulates immune cellular trafficking, and is crucial to angiogenesis. In brief, the endothelium is a major determinant of vascular homeostasis. It is easily damaged by intrinsic and extrinsic factors, and given its low proliferative potential and limited capacity for self-repair, is dependent on circulating bone marrow-derived EPC for regeneration. The extent to which the endothelial integrity (physical and functional) and bone marrow responsiveness is preserved mirrors cardiovascular health.

Endothelial dysfunction has been implicated as a central pathophysiological feature in a number of disease states. It is one of the earliest events in the pathophysiological process leading to atherosclerotic vascular disease [1], is identified as a central component in the pathogenesis of a diseases such as diabetes mellitus and sepsis [2,3], and more recently is associated with postoperative morbidity $[4,5 \bullet]$. Endothelial cell functions are sensitive to noxious stimuli [6], many of which are common to the perioperative period, including hypoxia (e.g., ischemia-reperfusion injury), exposure to 
inflammatory cytokines and endotoxins, and through direct injury from surgical manipulation or hemodynamic shear stress (Fig. 1). Injury alters the endothelial phenotype (with loss of the ability to synthesize and release mediators e.g., nitric oxide) tipping the balance of endothelial-derived factors to disrupt barrier function, enhance vasoconstriction, coagulation and leukocyte adhesion, and promote smooth muscle cell proliferation. These responses to injury are likely to exist as protective mechanisms, however, if the underlying basal endothelial reserve is limited, the injury severe or persistent, or the host response exaggerated, then the altered phenotype may result in loss of circulatory homeostasis, reduced tissue oxygen supply and end-organ dysfunction, culminating in an increased risk of postoperative complications.

This review explores endothelial dysfunction in the perioperative setting: improving our understanding of the role of endothelial dysfunction in postoperative morbidity, evaluating methods of assessing endothelial function as a strategy for improved preoperative risk stratification, and exploring the feasibility of modulating endothelial function to improve postoperative outcomes.

\section{Endothelial Dysfunction}

'Endothelial dysfunction', one of the earliest events in the pathophysiological process leading to atherosclerotic disorders [7], refers to a condition in which the endothelium loses its physiological properties: the tendency to promote vasodilation, fibrinolysis, and anti-aggregation.
Endothelial cells secrete multiple mediators that promote either vasodilation/anti-aggregation (nitric oxide, prostacyclin, carbon monoxide, endothelium-derived hyperpolarizing factor) or vasoconstriction/aggregation (endothelin-1, thromboxane-A2). The phenotypic expression of the endothelium can be seen as a dynamic 'set point' that ranges between a quiescent, an activated, or a dysfunctional state (Fig. 2). This set point reflects the balance between the underlying (chronic) health of the endothelium, acute exacerbating triggers such as inflammation and oxidative stress, and the 'regenerative' ability of the bone marrow $[8,9]$, which releases hematopoietic progenitors into the peripheral circulation.

Transient 'endothelial activation', with decreased bioavailability of homeostatic mediators such as nitric oxide, results in vasoconstriction, pro-inflammatory and prothrombotic changes. These changes serve as an adaptive physiologic response to acute stressors. This is illustrated by as much as $50 \%$ loss in endothelial vasodilator function within hours of ingesting a meal rich in saturated fat (described as a "Big Mac attack") [10]. The impact of diet on endothelial function is supported by a recent large prospective primary prevention outcomes study, which reported that in people at high cardiovascular risk, a Mediterranean diet supplemented with extra-virgin olive oil or nuts showed a $30 \%$ reduction in the incidence of major cardiovascular events when compared to a control diet (advice to reduce dietary fat) [11]. This is further illustrated in patients with type-2 diabetes mellitus, a disease characterized by endothelial dysfunction and impaired EPC mobilization [3], who have a two to four-fold
Fig. 1 The surgical proinflammatory and pro-oxidant milieu may result in both functional and structural alterations in the endothelium, resulting in hemostatic dysregulation and impaired local tissue perfusion, with consequent microvascular and macrovascular related postoperative complication (Illustration courtesy of Dr. Marissa Ferguson)

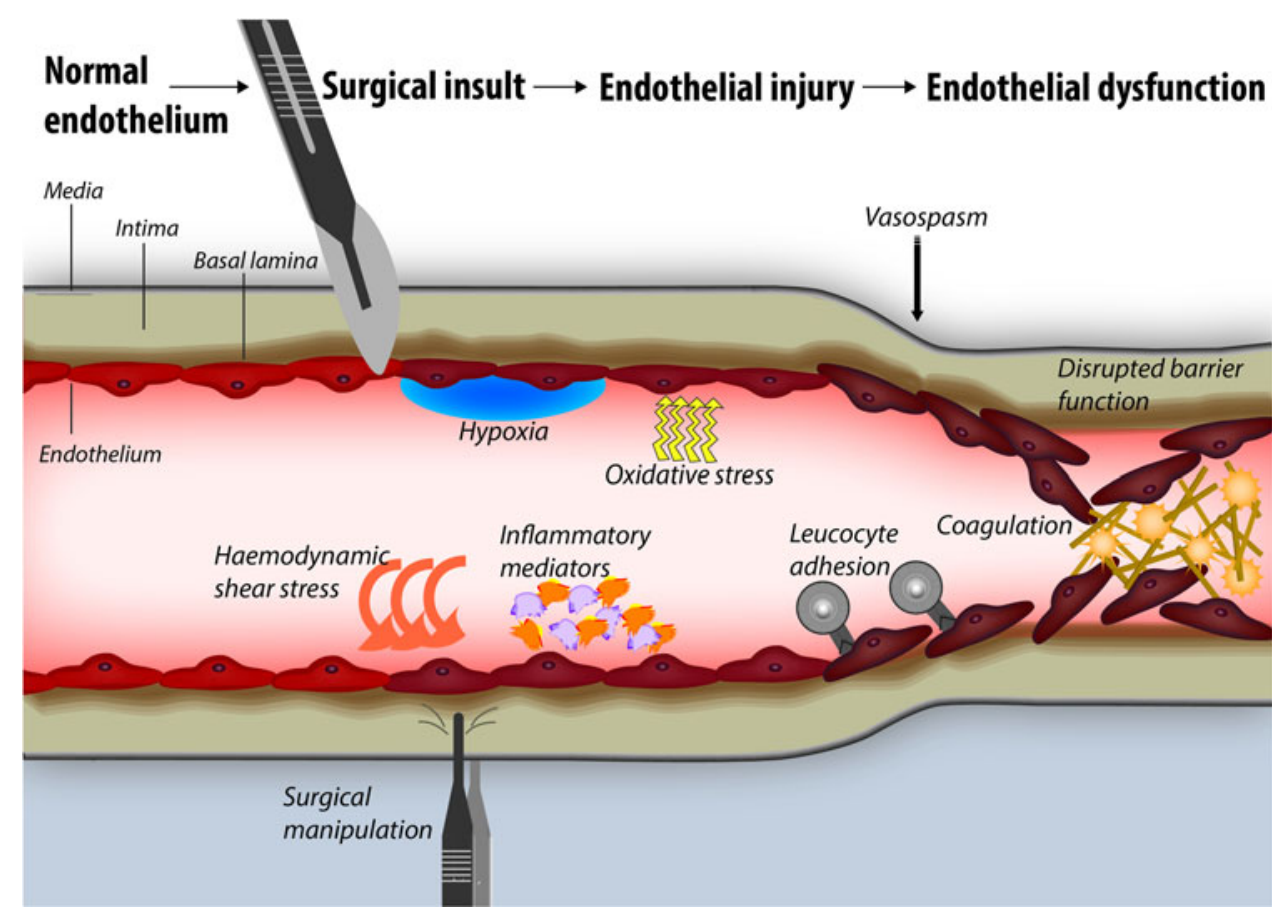




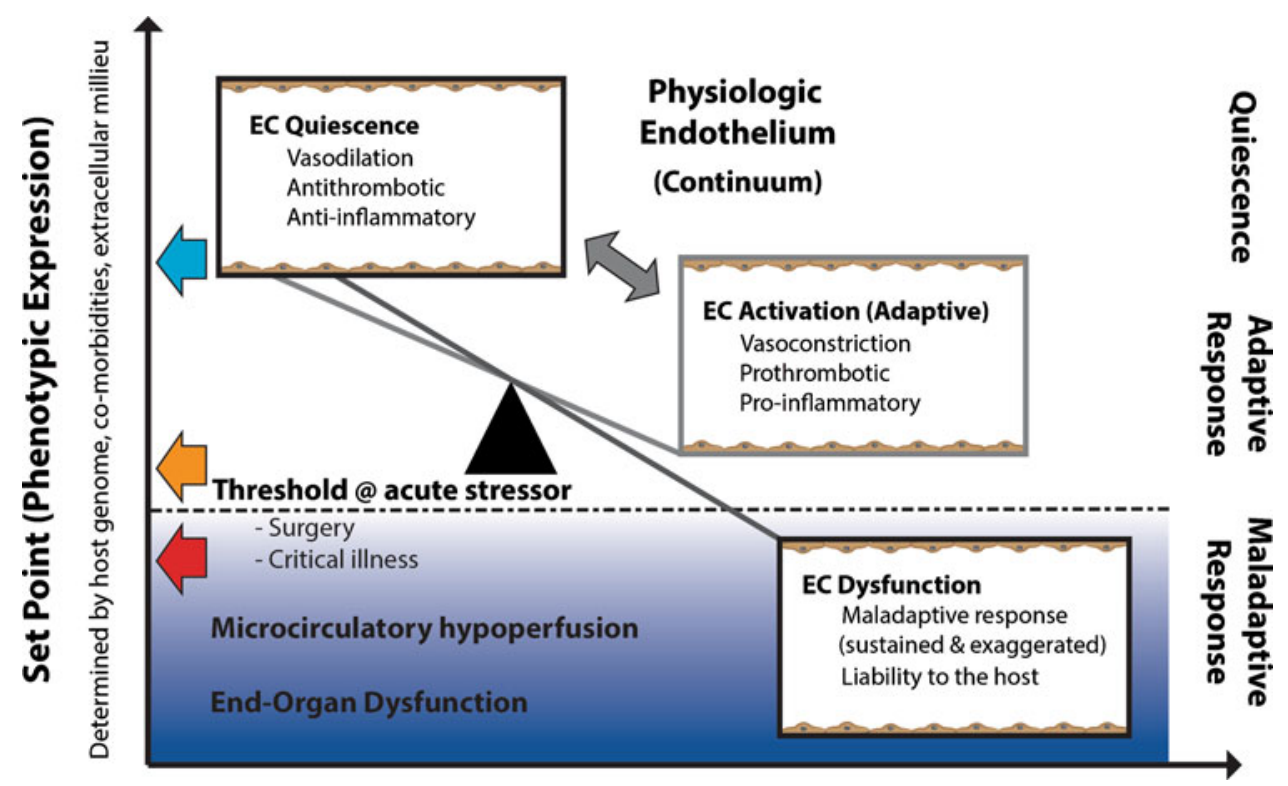

Fig. 2 The phenotypic expression of the endothelium can be described as a dynamic 'set point' that ranges between a quiescent, activated or dysfunctional state. This reflects on the balance between the underlying health of the endothelium, acute exacerbating triggers such as inflammation and oxidative stress, and the 'regenerative' ability of the bone marrow, which releases hematopoietic progenitors

increased risk of cardiovascular disease. As such, endothelial vasodilator dysfunction is a well-established surrogate that is predictive of cardiovascular morbidity and mortality [12].

\section{Pathophysiology of Endothelial Injury in the Perioperative Period}

Chronic exposure to cardiovascular risk factors and an exacerbated or persistent maladaptive response to acute pathophysiologic stressors, mediated through the inflammatory/oxidant burden and/or the prothrombotic effect of endothelial microparticles, impairs basal nitric oxide bioavailability - a hallmark of endothelial dysfunction [2, 13]. Endothelial dysfunction is likely mediated through an apoptotic process, with inflammatory/oxidant stressors signaling the MAPK and JNK pathways to suppress endothelial-cadherin, and consequent activation of the caspase protein family leading to intimal denudation through apoptosis [3]. This detachment of entire endothelial cells (circulating endothelial cells CEC) or apoptotic endothelial microparticles can be characterized and measured.

Inflammation is extremely injurious to the endothelium [14], and almost ubiquitous in the perioperative period to varying degrees. In human volunteers, a challenge with pro-inflammatory cytokines results in a transient loss of into the peripheral circulation. Inadequate basal endothelial function and/or a maladaptive host response to pathophysiologic changes that accompany surgical stress may result in deterioration of the endothelial reserve below a critical 'physiologic threshold' that is required to sustain microvascular perfusion

endothelial vasodilator function, with recovery occurring up to seven days after the inflammatory challenge [15]. Importantly, studies have shown reversibility to inflammation-induced endothelial dysfunction through source removal [16] or though anti-inflammatory strategies (hydrocortisone or high dose aspirin) [15, 17]. A significant improvement in endothelial vasodilator function was observed six months after aggressive treatment of periodontitis, however, important to the perioperative period, endothelial-dependent vasodilator function initially deteriorated in the acute period $(24 \mathrm{~h})$ after periodontal treatment [16]. These studies demonstrate a temporal link between an acute systemic inflammatory load and acute deterioration of endothelial function. This link is supported by a study that undertook serial measuring of endothelial function after surgery and found a reduction in endothelial function in the early postoperative period. Of note, decline in endothelial function was greater after laparotomy compared with laparoscopy, with recovery of endothelial function to baseline by the seventh postoperative day [18••]. Similarly, another study displayed a decline in endothelial function 24-48 $\mathrm{h}$ after cardiopulmonary bypass for coronary artery bypass surgery [19].

These observations provide a plausible explanation for the peak incidence of myocardial infarction occurring in the postoperative period, when flow stagnation and increased thrombogenicity prevails; [20] and for lower cardiovascular complication rates observed for laparoscopy 
compared with laparotomy [21]. The link between inflammation, endothelial dysfunction and vascular events is supported by the observation in population studies of a seasonal variation in myocardial infarction; with increased incidence in the winter months when inflammatory infections occur more frequently [22]. It is this combination of post-surgical inflammation, endothelial dysfunction, and a prothrombotic state that increases the risk of microvascular impairment and postoperative morbidity, and is likely to be more evident in patients with marginal preoperative (baseline) endothelial function [4, 23].

\section{Clinical Implications of Perioperative Endothelial Dysfunction}

Patients undergoing major surgery are at substantial risk for postoperative morbidity, with $30-60 \%$ of patients developing complications [24, 25]. Endothelial dysfunction in the perioperative period may contribute to the risk of postoperative complications via impaired vascular homeostasis and resultant reduced tissue perfusion.

Specifically, inadequate baseline (preoperative) endothelial function and/or a maladaptive host response to pathophysiologic changes accompanying surgical stress may result in deterioration of the endothelial reserve below a critical 'physiologic threshold' that is required to sustain microvascular perfusion (Fig. 2). As such, the surgical proinflammatory and pro-oxidant milieu may result in both functional and structural alterations in the endothelium, resulting in hemostatic dysregulation with impaired local tissue perfusion, and consequent micro- and macro-vascular related postoperative complications (Fig. 1) [4, 26].

Methods that characterize the underlying endothelial reserve (e.g., assess functionality through endothelialdependent vasodilation), quantify vascular insult (e.g., measure endothelial, thrombogenic and inflammatory biomarkers, and levels of denuded CECs or endothelial microparticles), and enumerate the functional regenerative capacity of EPCs have a growing role in our clinical armamentarium [27]. These tools are increasingly being explored as methods of improving preoperative risk stratification [4, 5, 23, 28, 29], improving our understanding of the pathophysiology of perioperative endothelial dysfunction [18••], and our understanding of potential therapeutic strategies [30]. As such, endothelial vasodilator dysfunction has been shown to be predictive of short and long-term postoperative cardiovascular events in patients undergoing vascular surgery [4]. Further, in a recent study of critically ill patients, we demonstrated that those patients with lower vascular reactivity, indicative of a worse systemic endothelial function, had increased duration of acute brain dysfunction (delirium and coma). This study points to a plausible relationship between alterations in cerebral blood flow and blood-brain barrier permeability and acute brain injury after critical illness (ICU-related neurocognitive dysfunction) [31•].

Preoperative assessment of endothelial function and reserve will likely be particularly useful for refining risk stratification in the increasing number of patients who present for major non-cardiac surgery and have multiple preoperative risk factors, including hypertension, diabetes, obesity, and dyslipidemia (otherwise termed the metabolic syndrome), yet are without overt symptoms or history of cardiovascular events. These patients are often classified as American Society of Anesthesiologists (ASA) Physical Class 2, thereby underestimating the propensity toward complications. In a large retrospective analysis of patients with metabolic syndrome, patients with diabetes, hypertension or obesity who underwent major joint replacement surgery were at greater risk of postoperative complications [32]. This increased risk for postoperative morbidity may be partly attributed to underlying endothelial dysfunction, as supported by the fact that patients with the metabolic syndrome have impaired endothelial function and decreased EPC levels [3]. Similarly, endothelial damage and microcirculatory impairment are early pathogenetic events in the end-organ damage (cardiomyopathy, nephropathy, retinopathy, and neuropathy) associated with diabetes, likely mediated through impaired mobilization, proliferation, survival and homing of EPCs resulting in reduced capillary density, increased fibrosis, and impaired end-organ function [3].

Apart from a diagnostic and prognostic role, the mature endothelium, and increasingly the hematopoietic progenitors (e.g., EPCs), also provide an attractive therapeutic target to stimulate angiogenesis, vasculogenesis and endothelial function [33]. Improved risk stratification and the opportunity to modulate the endothelial-thrombotic-inflammatory cascades will likely lead to improved perioperative outcomes. The importance of improved risk stratification and therapeutic modulation of endothelial function is reflected in the ubiquitous presence of the endothelium, whereby, in the opinion of the authors, underlying subclinical microvascular endothelial dysfunction is likely to have a greater impact on perioperative morbidity, contributing to complications such as impaired wound healing and end organ dysfunction, than the lower incidence of, albeit more devastating, macrovascular events.

\section{Noninvasive Assessment of Endothelial Vasodilator Function}

An indirect assessment of endothelial function can be obtained through examination of endothelial vasodilator influences in response to hyperemia [34]. The tractive force 
of fluid flow stimulates the endothelium to release vasodilators, most prominently nitric oxide. This phenomenon of induced vascular reactivity can be observed directly by ultrasound imaging of brachial artery diameter [34] or indirectly through monitoring of peripheral temperature change [35].

This technology has been widely utilized to document the association of endothelial vasodilator dysfunction with cardiovascular risk factors, the relationship of endothelial vasodilator dysfunction to various biomarkers, such as C-reactive protein, or asymmetric dimethylarginine (the endogenous antagonist of nitric oxide synthase), and the correction of endothelial vasodilator function with nutritional, therapeutic and lifestyle (exercise) modifications is technically more challenging and the reliability of the methods may be questionable in terms of their accuracy, sensitivity and specificity [36॰]. Moreover, these tests are surrogate markers of localized fibroelastic and kinetic endothelial function. However, with the endothelium being a ubiquitous organ, they are without true assessment of functional changes and the etiology at a cellular and biochemical level.

\section{Brachial Artery Reactivity Testing (BART)}

The most prevalent method used to assess endothelial function has been to assess the regulation of vascular reactivity non-invasively with duplex ultrasonography. This Brachial Artery Reactivity Test (BART) assesses flow-mediated dilation (FMD) and peak flow velocity in response to induced hyperemia (following limb occlusion through blood pressure cuff inflation to supra-systolic levels) using high-resolution ultrasound. This technique emerged as a clinical research tool for studying endothelium-dependent vasomotor function in the early 1990s [34]. FMD has developed as a noninvasive and broadly applicable method for examining endothelial function, and as an early surrogate of atherosclerosis that correlates well with both endothelial dysfunction in the coronary circulation and with overall cardiovascular outcomes [37, 38]. It is now a commonly used research tool to evaluate risk factor status and preclinical disease states, and to monitor improvement in endothelial function following targeted interventions and risk factor modifications [39]. However, despite its deceptively simple appearance, ultrasonographic assessment of brachial artery reactivity is technically challenging, requiring expertise and sensitive ultrasound equipment, and, hence, has restricted its use to expert vascular laboratories and research settings. In an attempt to standardize the techniques and allow more routine diagnostic use of BART, recent guidelines were published for the ultrasound assessment of endothelial-dependent FMD of the brachial artery [40].
FMD has been found to improve non-invasive preoperative risk stratification in patients scheduled for vascular surgery, with impaired brachial artery endothelial function independently predicting postoperative cardiac events [4]. Similarly, in a recent clinical study among major thoracic surgical patients, we demonstrated that BART was a good predictor of postoperative morbidity and mortality [5•]. Larger studies are required to determine whether FMD can provide further insight into the pathophysiology of endothelial dysfunction or contribute to day to day risk-stratification and prognostication preoperatively to guide clinical decision-making, requires larger studies [29].

\section{Digital Thermal Monitoring (DTM)}

Digital thermal monitoring (DTM), a novel and non-invasive FDA approved method is currently under evaluation in clinical trials for the assessment of peripheral vascular function, and improvement of cardiovascular risk assessment $[35,37]$. This method indirectly measures changes in skin blood flow following reactive hyperemia, utilizing a thermo-coupling method to measure temperature. A recent study showed that the DTM measured temperature rebound closely correlates with Doppler flow velocity [41]. We observed a lower DTM signal in patients with cardiovascular risk factors (abdominal obesity, smoking) and this may contribute to further risk stratification of these patients. However, unlike the findings of $\mathrm{Hu}$ et al. [18••], we were unable to show significant DTM changes in the perioperative period following major thoracic surgery [23].

\section{Peripheral Artery Tonometry (EndoPAT)}

This novel technique measures peripheral vasodilator response using fingertip pulse amplitude tonometry for non-invasive assessment of vascular function. The technique is based on a system of inflatable latex air cuffs placed on the middle finger and connected by pneumatic tubes to an inflating device. Using a constant counter pressure through the air cushions on the finger to prevent venous pooling, venoarteriolar reflex vasoconstriction and occlusion of arterial blood flow, the device senses pulsatile volume changes of the distal digit induced by pressure alterations in the finger cuffs [42]. Impairment of pulse amplitude hyperemic response has been demonstrated among patients with coronary artery endothelial dysfunction [43] and with multiple traditional metabolic risk factors [38].

However, whether predominantly microvascular hyperemic responses, as measured at the fingertip by DTM or EndoPAT techniques, correlates with the gold standard of brachial artery FMD, a macrovascular measure, remains contentious. One study investigated pulse wave form 
analysis and refuted the claims that large (macrovascular) and small (microvascular) arterial stiffness are substitute measures for sonographic assessments of brachial FMD [44]. Moreover, whether either of these non-invasive techniques are a useful clinical tool, in order to refine preoperative patient risk assessment and care, has yet to be investigated in large clinical trials.

\section{Plasma Biomarkers of Endothelial Dysfunction}

The shift of the normal endothelium to a damaged, procoagulant, pro-inflammatory, vasoconstricted state, with decreased regenerative and reparative capacity, can occur in chronic disease states, in cancer, after inflammatory insults, and after major surgery. Functional assessment, e.g., FMD using non-invasive techniques, or assessment of inflammatory markers, such as CRP, can demonstrate this status, but are relatively insensitive and non-specific. They fail to provide a dynamic assessment of this functional environment, nor information regarding the pathophysiology of endothelial dysfunction. There is a need to explore more sensitive and specific biomarkers that can be utilized real-time in routine diagnostic laboratories. Biochemical and cellular biomarkers of endothelial (dys)function, (such as dimethyl arginine levels, markers of lipid peroxidation, inflammatory mediators, P-selectin, endothelial microparticles, indices of coagulation, and cellular surrogates such as microparticles, CEC, and circulating EPC) may provide greater insight into the mechanistic process and thus provide better risk prediction and guidance of endothelial function and optimization strategies [3, 45]. A number of these biomarkers are currently under investigation in various disease states, including surgical populations [46, 47], but is beyond the scope of this review. Many of these biomarkers are still considered investigational and only available in highly specialized laboratories. An area of developing interest lies in endothelial microparticles for their diagnostic and prognostic value [46, 47], and in EPC mobilization and functionality for their prognostic $[9,48]$ and therapeutic capacity [49••].

\section{Perioperative Therapeutic Strategies to Improve Endothelial Function}

Current clinical strategies applicable to the perioperative setting that may modulate endothelial dysfunction or preserve microvascular health are centered upon reduced inflammatory burden and/or up-regulation of endothelial nitric oxide synthase. These strategies are aimed at preserving NO bioavailability and include: preoperative exercise therapy, avoidance of drug withdrawal (e.g., aspirin, statins) [50], anti-inflammatory medications (e.g., aspirin, statins), and other pharmacologic interventions (e.g., newer NO-enhancing $\beta$-blockers). Many of these strategies are still in their infancy and large prospective trials investigating the impact of these therapeutic options on postoperative outcome are eagerly awaited, particularly in patients identified preoperatively with endothelial dysfunction. Data supporting interventional strategies include: studies that have shown reversibility of inflammation-induced endothelial dysfunction through source removal [16] or though antiinflammatory strategies including hydrocortisone, aspirin and statin therapy $[15,19]$.

\section{Pharmacological Intervention: Statin Therapy}

Statins are indicated for primary or secondary prevention of cardiac events [51]. In addition to lowering cholesterol, numerous studies have demonstrated benefit through their pleiotropic effects, which include strong anti-inflammatory effects and improved endothelial function, including EPC mobilization. These effects lead to reduced coronary artery plaque formation and stabilization of existing coronary plaques [52, 53], contributing to a reduction of short-term and long-term cardiovascular complications and death.

A randomized placebo controlled clinical trial showed that preoperative statin use for a median of 37 days before non-cardiac vascular surgery significantly lowered the incidence of myocardial ischemia (statin, $10.8 \%$ vs. placebo, $19 \%$ ), myocardial infarction (statin, $4.8 \%$ vs. placebo, $10.1 \%$ ), and cardiac death [54]. Interleukin-6 and high-sensitive C-reactive protein levels were lower in patients randomized to statin treatment. Meta-analyses of studies investigating the effect of statin therapy on postoperative outcome suggest that statin administration is associated with decreased postoperative cardiac events [55-57]. A recent meta-analysis of preoperative statin therapy in cardiac surgery reported a $0.9 \%$ absolute risk (2.6 vs. $3.5 \%$ ) and $31 \%$ odds reduction for early all-cause mortality with preoperative statin use [58••]. In addition, postoperative atrial fibrillation, stroke, intensive care unit and in-hospital stay were also substantially reduced. In a randomized study, cardiac surgery patients assigned to preoperative statin therapy exhibited less decline in endothelium-dependent FMD $(60.1+15 \%$ decline in the placebo group compared with $45.8+16.6 \%$ in the atorvastatin group; $p<0.05$ ) after cardiopulmonary bypass [19]. These data support the endothelial protective benefit attributed to statins. Unfortunately, some of the literature base has questionable scientific validity due to that published by a discredited researcher $[59,60]$.

A withdrawal effect for statins, with increased risk of postoperative cardiac complications, suggests that patients 
on chronic statin treatment should continue taking statins in the perioperative period to prevent such adverse outcome [61]. In patients with no history of prior statin use but with multiple cardiac risk factors and elevated levels of inflammatory markers (interleukin-6, and C-reactive protein) initiation of statin use at least 30 days prior to the planned surgical procedure could be considered [62], however, a shorter duration may be feasible as a rapid physiological effect was demonstrated in a prospective trial where a single oral dose of pravastatin (40 mg) significantly attenuated acetylcholine-mediated vasoconstriction after $24 \mathrm{~h}$ [63].

\section{Cellular Aspects: Endothelial Progenitor Cells}

Endothelial dysfunction can be improved by the reconstitution of the endothelial layer, which generally involves the biologic paradigms often described as angiogenesis and vasculogenesis. Angiogenesis refers to the neovascularization occurring via migration and proliferation of endothelial cells of pre-existing vessels. The capacity of mature endothelial cells to proliferate, however, depends on the presence of endothelial colony forming cells (CFC) that give rise to endothelial progeny [64-66]. Since there might be heterogeneity in the level of proliferative potential, for example depending on the vascular bed, adequate vascular repair might require additional support. In this context, adult vasculogenesis - the de novo formation of blood vessels from EPC - has been demonstrated to play an important role.

The presence of circulating blood cells with the ability to promote vascular repair and regeneration was first described in 1997 [8]. These identified cells displayed a variety of seemingly endothelial-specific cell surface antigens, and were therefore referred to as EPC. Subsequently, an accumulation of experimental studies have been performed to assess the mechanism by which bone marrowderived EPC may be recruited and incorporated into sites of active neovascularization during tissue ischemia, vascular trauma, tumor growth and inflammation. In parallel, a multitude of clinical studies have identified EPC as a biomarker for clinical disorders such as cardiovascular disease [9], cerebrovascular disease [67, 68], sepsis [48], and numerous types of cancer $[69,70]$. In all of these studies, the concentration of circulating EPC inversely correlates with the risk of adverse outcome. Subsequently, experimental data from marrow transplantation have shown that marrow-derived cells are recruited to sites of active neovascularization and can differentiate into vascular cells in situ. The frequency of this phenomenon and the identification of the cell type involved are yet to be fully determined [71].
Different Populations of Endothelial Progenitor Cells

A major limiting factor in this field has been the lack of a specific marker to identify circulating EPCs. Furthermore, different methods have been applied to detect EPCs (flow cytometry, culture methods, immunostaining), rendering comparison difficult. However, the definition of three functional populations of EPCs has been generally accepted. The first population expresses the phenotype CD34+ $\mathrm{AC} 133+\mathrm{KDR}+$ and has gained wide use as a means to measure circulating EPC in human subjects [72]. These cells may be recruited to denuded vessels in ischemic sites, but they do not directly become persistent vascular endothelial cells or display de novo in vivo vasculogenic potential. Rather, they display potent paracrine properties regulating new vessel formation via angiogenesis [73, 74]. Yoder et al. [33, 64, 75] refer to these cells as proangiogenic hematopoietic cells.

Other populations of EPC are identified using colonyforming assays, in which plated human CD34+ peripheral blood cells form cellular clusters on fibronectin-coated dishes in vitro. Ashara et al. [8] described these clusters binding acLDL in the initial study as evidenced by CD34+ peripheral blood cells differentiating into spindleshaped endothelial cells. The emerging cell clusters are referred to as EPC colony forming units. The third population of EPCs has been identified as yet another type of cell colony emerging from plated peripheral blood mononuclear cells. This cell colony emerges as tightly adherent with a typical cobblestone appearance and is referred to as endothelial colony forming cells (ECFC), late outgrowth cells (OEC) or blood outgrowth endothelial cells (BOEC). These cells have vessel-forming ability, but also connect to the vessels to become part of the systemic circulation of the host animal [76]. Among all current putative EPCs, ECFCs appear to function as a circulating precursor with in vivo human vessel-forming ability and exhibits the most features consistent with human postnatal vasculogenic cells.

EPC enumeration has been correlated with cardiovascular risk factors, extent of coronary disease, and future cardiovascular events [9]. Given that EPC enumeration and functional characterization represent the only assessment of the reparative side of the balance between damage and regeneration, this technique may offer independent and different assessment of the propensity to cardiovascular injury, greatly improving risk stratification of patients. Attempts to stimulate mobilization and homing of bone marrow derived EPC or exogenously administered cellbased (progenitor) therapy will likely also emerge in the next decade as peripherally circulating EPCs and intrinsic stem cells play an important role in accelerating endothelialization and tissue remodeling at areas of vascular 
damage in both disease, and following toxic insults and stress [49••, 77, 78].

Comorbid disease states and aging are associated with decreased regenerative ability by EPCs and may account for increased risk of postoperative complications and delayed recovery. As such, diabetes is characterized by weak bone marrow mobilization, decreased proliferation, and shortened survival of EPC [3]. Inhibition of oxidant stress normalizes post-ischemic neovascularization in diabetes by positive EPC modulation. Bone marrow EPC mobilization was partially rescued in diabetic rats treated with insulin. It is not known whether this favorable effect is mediated by insulin itself or by improved glucose control.

\section{Mobilization of Endothelial Progenitor Cells}

Many factors have been described as having important roles in the mobilization of EPCs [79, 80]. Among them are growth factors (such as VEGF, placental growth factor, erythropoietin, and angiopoietin-1), pro-inflammatory cytokines, growth factors (such as GM-CSF and GCSF), chemokines (such as stromal cell-derived factor-1), hormones (such as estrogens, and lipid-lowering and antidiabetic drugs), as well as physical activity [81]. The stimulatory effect of exercise on EPC has been shown not only in highly trained athletes [82] and healthy subjects [83], but also in patients with known cardiovascular disease [84]. However, little is known about the benefit of exercise to endothelial health in patients with subclinical cardiovascular disease that are predisposed to endothelial dysfunction due to comorbidities including metabolic syndrome or in patients subjected to the acute inflammatory insult around the time of surgery.

Prehabilitation, with an exercise regimen, has been shown to improve exercise capacity (anerobic threshold and $\mathrm{pVO}_{2}$ ) and endothelial reserve. The duration and intensity of exercise needed to adequately stimulate EPC is still unclear. In a study with healthy subjects, Laufs et al. [83] reported increased circulating EPC levels after moderate and intense running for $30 \mathrm{~min}$ (80-100\% velocity of individuals' anerobic threshold), but not after short term running for $10 \mathrm{~min}$. In elderly patients with documented coronary artery disease, a 4-week exercise program resulted in an up-regulation of circulating EPCs. More recently, a study showed that even a short (15-days) cardiac rehabilitation program caused an increase in EPCs in relation to improved exercise capacity. A 3-month cardiac rehabilitation program increased EPCs 2-fold, colony-forming units 3-fold, increased blood nitrite concentrations, and reduced EPC apoptosis [85]. Despite the information provided by these studies it remains to be determined what minimum threshold of training duration and intensity is required to elicit improvements in endothelial function[86] and whether such prehabilitation strategies, prior to surgery, will reduce perioperative morbidity.

Surgical injury induces the mobilization of EPCs, with significantly higher circulating EPC and bone marrow EPC levels observed $24 \mathrm{~h}$ after surgery in an animal model [87]. The ability to mount an EPC response is also seen in critical illness, and the response is significantly greater in patients that survive sepsis [48], and recover from illness, e.g., without fibrotic changes after pneumonia [77].

Given that 'responders' who mount a 'cellular' stress response to injury, with increased EPC mobilization, have improved organ recovery [77] and improved survival [48], it is intriguing as to the necessity of a bone marrow-derived cellular component to the 'stress response' and whether strategies to improve bone marrow capacity and responsiveness will influence a patient's ability to withstand surgical injury. Increasing this bone marrow-derived regenerative response through preoperative exercise training may be a potential therapeutic option to optimize patients prior to surgery. In a recent pilot study, we were able to demonstrate that patients scheduled for major thoracic surgery that exhibited an EPC response to the stressor of preoperative exhaustive exercise suffered significantly fewer postoperative complications (manuscript under review).

However, discovering an inadequate EPC response during acute illness, such as impaired wound healing, pneumonia, acute lung injury [45] or sepsis [88] is too late. Using a surrogate stressor, e.g., exercise and prehabilitating patients prior to surgery to improve bone marrow responsiveness is therefore appealing. Importantly, some of the endothelial dysfunction, particularly that acquired in the perioperative period, may be transient or reversible and may not actually involve structural change in the cells of the vascular endothelium, but more likely potentially reversible alterations in function-so these wouldn't require new cells, just repair of a damaged process.

Importantly, whether this lack of EPC response is an epiphenomenon, a surrogate marker, or indeed causative of increased postoperative complications, requires further study. The causative nature is supported by animal studies that suggest that exogenous EPC administration can rescue endotoxin-induced ARDS, with reduced inflammation, improved oxygenation, and improved survival [49••, 78]. Similarly, Jeong et al. [89] investigated whether diabetic neuropathy could be reversed by local transplantation of EPCs. They found that motor and sensory nerve conduction velocities, blood flow, and capillary density were reduced in sciatic nerves of streptozotocin-induced diabetic mice, but recovered to normal levels after hind-limb injection of bone marrow-derived EPCs. Injected EPCs were preferentially and durably engrafted in the sciatic nerves. Finally, they found that portions of engrafted EPCs were uniquely localized in close proximity to vasa nervorum. This study shows, 
for the first time, that bone marrow-derived EPCs could reverse various manifestations of diabetic neuropathy. As such, cell-based translational approaches may provide a novel and valid therapeutic alternative in the future.

Exercise [90] and tissue insult from surgery [87] are known to increase the mobilization of EPC. In this manner, cardiopulmonary exercise testing (CPET) can be used as a catalyst to increase the circulating population of EPCs and as an evaluation tool of a patient's ability to mount an EPC response preoperatively. Unlike Laufs et al., who exercised patients to a moderate intensity, the use of CPET, with exercise to maximum capacity, not only increases the EPC population, but also provides incremental information about the capacity of EPC release from the bone marrow in response to a stressor similar to the surgical stress. Additional gas exchange parameters obtained in a diagnostic CPET (anerobic threshold and peak $\mathrm{VO}_{2}$ ) can be used to determine patients' individual physiologic capacity and the amount of exercise needed in order to stimulate the population of EPC. Preoperatively, exercise training could be used to condition patients' individual capacity and to improve endothelial function by affecting EPC number and function. As such, Cesari et al. [81] reported a significant increase in circulating EPCs in those patients that showed an improvement of exercise capacity of more than $23 \%$ (as measured by the six minute walk test) after completion of a rehabilitation program.

\section{Conclusion}

The clinical utility of noninvasive methods that assess preoperative endothelial/microvascular (dys)function and bone marrow reserve will reflect upon the concerted interactions of the endothelial, inflammatory and thrombotic cascades and likely expand and contribute to improved risk stratification, thereby allowing opportunity for timely perioperative optimization. In this regard, there is increasing recognition of the need for perioperative strategies that maintain the integrity of the endotheliumpreserving homeostasis within the endothelial-inflammatory-coagulation/fibrinolytic (hemostatic) cascades-and for strategies that preserve, modulate, or repair endothelial function. Targeting the endothelium and maximizing inherent physiological function in the perioperative period may improve postoperative outcomes.

Disclosure Bernhard Riedel declares that he has no conflict of interest. Neysan Rafat declares that he has no conflict of interest. Kim Browne declares that she has no conflict of interest. Kate Burbury is supported by a grant from Covidien (research study for thrombogenic biomarkers in cancer patients). Robert Schier declares that he has no conflict of interest.
Compliance with Ethics Guidelines This article does not contain any studies with human or animal subjects performed by any of the authors.

\section{References}

Papers of particular interest, published recently, have been highlighted as:

- Of importance

•• Of major importance

1. Tousoulis D, Charakida M, Stefanadis C. Endothelial function and inflammation in coronary artery disease. Heart. 2006;92: $441-4$.

2. Cepinskas G, Wilson JX. Inflammatory response in microvascular endothelium in sepsis: role of oxidants. J Clin Biochem Nutr. 2008;42:175-84.

3. Avogaro A, Albiero M, Menegazzo L, de Kreutzenberg S, Fadini GP. Endothelial dysfunction in diabetes: the role of reparatory mechanisms. Diabetes Care. 2011;34(Suppl 2):S285-90.

4. Gokce N, Keaney JF, Hunter LM, Watkins MT, Menzoian JO, Vita JA. Risk stratification for postoperative cardiovascular events via noninvasive assessment of endothelial function: a prospective study. Circulation. 2002;105:1567-72.

5. - Schier R, Hinkelbein J, Marcus H, et al. Preoperative microvascular dysfunction: a prospective, observational study expanding risk assessment strategies in major thoracic surgery. Ann Thorac Surg. 2012;94:226-33. This study highlights the role of improved perioperative risk stratification by testing for endothelial vasodilator function.

6. Bhagat K, Moss R, Collier J, Vallance P. Endothelial "stunning" following a brief exposure to endotoxin: a mechanism to link infection and infarction? Cardiovasc Res. 1996;32:822-9.

7. Davies PF. Endothelial mechanisms of flow-mediated atheroprotection and susceptibility. Circ Res. 2007;101:10-2.

8. Asahara T, Murohara T, Sullivan A, et al. Isolation of putative progenitor endothelial cells for angiogenesis. Science. 1997; 275:964-7.

9. Hill JM, Zalos G, Halcox JPJ, et al. Circulating endothelial progenitor cells, vascular function, and cardiovascular risk. N Engl J Med. 2003;348:593-600.

10. Vogel RA. Eating, vascular biology, and atherosclerosis: a lot to chew on. Eur Heart J. 2006;27:13-4.

11. Estruch R, Ros E, Salas-Salvado J, et al. Primary prevention of cardiovascular disease with a Mediterranean diet. N Engl J Med. 2013;368:1279-90.

12. Schachinger V, Britten MB, Zeiher AM. Prognostic impact of coronary vasodilator dysfunction on adverse long-term outcome of coronary heart disease. Circulation. 2000;101:1899-906.

13. Amar S, Gokce N, Morgan S, Loukideli M, Van Dyke TE, Vita JA. Periodontal disease is associated with brachial artery endothelial dysfunction and systemic inflammation. Arterioscler Thromb Vasc Biol. 2003;23:1245-9.

14. Clapp BR, Hingorani AD, Kharbanda RK, et al. Inflammationinduced endothelial dysfunction involves reduced nitric oxide bioavailability and increased oxidant stress. Cardiovasc Res. 2004;64:172-8.

15. Bhagat K, Vallance P. Inflammatory cytokines impair endothelium-dependent dilatation in human veins in vivo. Circulation. 1997;96:3042-7. 
16. Tonetti MS, D'Aiuto F, Nibali L, et al. Treatment of periodontitis and endothelial function. N Engl J Med. 2007;356:911-20.

17. Farb MG, Tiwari S, Karki S, et al. Cyclooxygenase inhibition improves endothelial vasomotor dysfunction of visceral adipose arterioles in human obesity. Obesity. 2013; doi:10.1002/oby. 20505.

18. • Hu YJ, Wei AN, Chook P, et al. The impact of noncardiovascular surgery on reactive hyperaemia and arterial endothelial function. Clinical and experimental pharmacology \& physiology. 2013. This study highlights the decline in endothelial vasodilator function during the postoperative period.

19. Chello M, Goffredo C, Patti G, et al. Effects of atorvastatin on arterial endothelial function in coronary bypass surgery. Eur $\mathrm{J}$ Cardiothorac Surg. 2005;28:805-10.

20. Biccard BM, Rodseth RN. The pathophysiology of peri-operative myocardial infarction. Anaesthesia. 2010;65:733-41.

21. Kikura M, Oikawa F, Yamamoto K, et al. Myocardial infarction and cerebrovascular accident following non-cardiac surgery: differences in postoperative temporal distribution and risk factors. J Thromb Haemost. 2008;6:742-8.

22. Vallance $\mathrm{P}$, Collier J, Bhagat $\mathrm{K}$. Infection, inflammation, and infarction: does acute endothelial dysfunction provide a link? Lancet. 1997;349:1391-2.

23. Schier R, Marcus HE, Mansur E, et al. Evaluation of digital thermal monitoring as a tool to assess perioperative vascular reactivity. J Atheroscler Thromb. 2013;20:277-86.

24. Licker M, Spiliopoulos A, Frey JG, et al. Risk factors for early mortality and major complications following pneumonectomy for non-small cell carcinoma of the lung. Chest. 2002;121:1890-7.

25. Duque JL, Ramos G, Castrodeza J, et al. Early complications in surgical treatment of lung cancer: a prospective, multicenter study. Grupo Cooperativo de Carcinoma Broncogenico de la Sociedad Espanola de Neumologia y Cirugia Toracica. Ann Thorac Surg. 1997;63:944-50.

26. Riedel B, Schier R. Endothelial dysfunction in the perioperative setting. Semin Cardiothorac Vasc Anesth. 2010;14:41-3.

27. Gokce N. Clinical assessment of endothelial function: ready for prime time? Circ Cardiovasc Imaging. 2011;4:348-50.

28. Gokce N, Keaney J F Jr, Hunter LM, et al. Predictive value of noninvasively determined endothelial dysfunction for long-term cardiovascular events in patients with peripheral vascular disease. J Am Coll Cardiol. 2003;41:1769-75.

29. Schier R, Riedel B. Brachial artery reactivity testing for preoperative microvascular risk assessment in major thoracic surgery. Ann Thorac Surg. 2013;95:1140.

30. Hindler K, Shaw AD, Samuels J, Fulton S, Collard CD, Riedel B. Improved postoperative outcomes associated with preoperative statin therapy. Anesthesiology. 2006;105:1260-72 Quiz 89-90.

31. - Hughes CG, Morandi A, Girard TD, et al. Association between endothelial dysfunction and acute brain dysfunction during critical illness. Anesthesiology. 2013;118:631-9. This study provides early insight into a plausible link that may exist between critical illness and endothelial dysfunction, with contribution of alterations of cerebral blood flow and blood-brain barrier permeability to acute brain dysfunction of critical illness.

32. Jain NB, Guller U, Pietrobon R, Bond TK, Higgins LD. Comorbidities increase complication rates in patients having arthroplasty. Clin Orthop Relat Res. 2005:232-8.

33. Richardson MR, Yoder MC. Endothelial progenitor cells: quo vadis? J Mol Cell Cardiol. 2011;50:266-72.

34. Celermajer DS, Sorensen KE, Gooch VM, et al. Non-invasive detection of endothelial dysfunction in children and adults at risk of atherosclerosis. Lancet. 1992;340:1111-5.

35. Gul KM, Ahmadi N, Wang Z, et al. Digital thermal monitoring of vascular function: a novel tool to improve cardiovascular risk assessment. Vasc Med. 2009;14:143-8.
36. - Flammer AJ, Anderson T, Celermajer DS, et al. The assessment of endothelial function: from research into clinical practice. Circulation. 2012;126:753-67. A review of clinical applicability of assessing endothelial function.

37. Ahmadi N, Usman N, Shim J, et al. Vascular dysfunction measured by fingertip thermal monitoring is associated with the extent of myocardial perfusion defect. J Nucl Cardiol. 2009; $16: 431-9$.

38. Hamburg NM, Keyes MJ, Larson MG, et al. Cross-sectional relations of digital vascular function to cardiovascular risk factors in the Framingham Heart Study. Circulation. 2008;117: 2467-74.

39. Corretti M. Brachial artery reactivity: clinical tool or research toy? J Am Soc Echocardiogr. 2004;17:693-6.

40. Corretti MC, Anderson TJ, Benjamin EJ, et al. Guidelines for the ultrasound assessment of endothelial-dependent flow-mediated vasodilation of the brachial artery: a report of the International Brachial Artery Reactivity Task Force. J Am Coll Cardiol. 2002;39:257-65.

41. McQuilkin GL, Panthagani D, Metcalfe RW, et al. Digital thermal monitoring (DTM) of vascular reactivity closely correlates with Doppler flow velocity. Conf Proc IEEE Eng Med Biol Soc. 2009;1:1100-3.

42. Rubinshtein R, Kuvin JT, Soffler M, et al. Assessment of endothelial function by non-invasive peripheral arterial tonometry predicts late cardiovascular adverse events. Eur Heart J. 2010;31: $1142-8$.

43. Bonetti PO, Pumper GM, Higano ST, Holmes D R Jr, Kuvin JT, Lerman A. Noninvasive identification of patients with early coronary atherosclerosis by assessment of digital reactive hyperemia. J Am Coll Cardiol. 2004;44:2137-41.

44. Wright CI, Scholten HJ, Schilder JC, et al. Arterial stiffness, endothelial function and microcirculatory reactivity in healthy young males. Clin Physiol Funct Imaging. 2008;. doi:10.1111/j. 1475-097X.2008.00807.x.

45. Burnham EL, Taylor WR, Quyyumi AA, Rojas M, Brigham KL, Moss M. Increased circulating endothelial progenitor cells are associated with survival in acute lung injury. Am J Respir Crit Care Med. 2005;172:854-60.

46. Burger D, Schock S, Thompson CS, Montezano AC, Hakim AM, Touyz RM. Microparticles: biomarkers and beyond. Clin Sci. 2013;124:423-41.

47. Burger D, Touyz RM. Cellular biomarkers of endothelial health: microparticles, endothelial progenitor cells, and circulating endothelial cells. J Am Soc Hypertens. 2012;6:85-99.

48. Rafat N, Hanusch C, Brinkkoetter PT, et al. Increased circulating endothelial progenitor cells in septic patients: correlation with survival. Crit Care Med. 2007;35:1677-84.

49. • Cao JP, He XY, Xu HT, Zou Z, Shi XY. Autologous transplantation of peripheral blood-derived circulating endothelial progenitor cells attenuates endotoxin-induced acute lung injury in rabbits by direct endothelial repair and indirect immunomodulation. Anesthesiology. 2012;116:1278-87. This study highlights the regenerative role of the bone marrow and potential for a future role for exogenous cellular therapy.

50. Rebound risk: aspirin and statin withdrawal. Consumer reports. 2005;70:48.

51. Shepherd J, Cobbe SM, Ford I, et al. Prevention of coronary heart disease with pravastatin in men with hypercholesterolemia. West of Scotland Coronary Prevention Study Group. N Engl J Med. 1995;333:1301-7.

52. Downs JR, Clearfield M, Weis S, et al. Primary prevention of acute coronary events with lovastatin in men and women with average cholesterol levels: results of AFCAPS/TexCAPS. Air Force/Texas Coronary Atherosclerosis Prevention Study. JAMA. 1998;279:1615-22. 
53. Sacks FM, Pfeffer MA, Moye LA, et al. The effect of pravastatin on coronary events after myocardial infarction in patients with average cholesterol levels. Cholesterol and recurrent events trial investigators. N Engl J Med. 1996;335:1001-9.

54. Schouten O, Boersma E, Hoeks SE, et al. Fluvastatin and perioperative events in patients undergoing vascular surgery. $\mathrm{N}$ Engl J Med. 2009;361:980-9.

55. Poldermans D, Bax JJ, Kertai MD, et al. Statins are associated with a reduced incidence of perioperative mortality in patients undergoing major noncardiac vascular surgery. Circulation. 2003; 107:1848-51.

56. Kertai MD, Boersma E, Westerhout CM, et al. A combination of statins and beta-blockers is independently associated with a reduction in the incidence of perioperative mortality and nonfatal myocardial infarction in patients undergoing abdominal aortic aneurysm surgery. Eur J Vasc Endovasc Surg. 2004;28:343-52.

57. Lindenauer PK, Pekow P, Wang K, Gutierrez B, Benjamin EM. Lipid-lowering therapy and in-hospital mortality following major noncardiac surgery. JAMA. 2004;291:2092-9.

58. • Kuhn EW, Liakopoulos OJ, Stange S, et al. Preoperative statin therapy in cardiac surgery: a meta-analysis of 90,000 patients. Eur J Cardiothorac Surg. 2013. This meta-analysis highlights the clinical role of perioperative statin therapy, which is mediated through pleiotropic effects that include improved endothelial function and anti-inflammatory effects.

59. Bonnet F, Samama CM. Cases of fraud in publications: from Darsee to Poldermans. Presse medicale. 2012;41:816-20.

60. Poldermans D. Scientific fraud or a rush to judgment? Am J Med. 2013;126:e5-6.

61. Le Manach Y, Coriat P, Collard CD, Riedel B. Statin therapy within the perioperative period. Anesthesiology. 2008;108:1141-6.

62. Poldermans D, Bax JJ, Boersma E, et al. Guidelines for preoperative cardiac risk assessment and perioperative cardiac management in non-cardiac surgery: the Task Force for preoperative cardiac risk assessment and perioperative cardiac management in non-cardiac surgery of the European Society of Cardiology (ESC) and endorsed by the European Society of Anaesthesiology (ESA). Eur Heart J. 2009;30:2769-812.

63. Qaseem A, Snow V, Fitterman N, et al. Risk assessment for and strategies to reduce perioperative pulmonary complications for patients undergoing noncardiothoracic surgery: a guideline from the American College of Physicians. Ann Intern Med. 2006;144:575-80.

64. Yoder MC, Mead LE, Prater D, et al. Redefining endothelial progenitor cells via clonal analysis and hematopoietic stem/progenitor cell principals. Blood. 2007;109:1801-9.

65. Ingram DA, Mead LE, Moore DB, Woodard W, Fenoglio A, Yoder MC. Vessel wall-derived endothelial cells rapidly proliferate because they contain a complete hierarchy of endothelial progenitor cells. Blood. 2005;105:2783-6.

66. Ingram DA, Mead LE, Tanaka $\mathrm{H}$, et al. Identification of a novel hierarchy of endothelial progenitor cells using human peripheral and umbilical cord blood. Blood. 2004;104:2752-60.

67. Ghani U, Shuaib A, Salam A, et al. Endothelial progenitor cells during cerebrovascular disease. Stroke. 2005;36:151-3.

68. Rafat N, Beck G, Pena-Tapia PG, Schmiedek P, Vajkoczy P. Increased levels of circulating endothelial progenitor cells in patients with Moyamoya disease. Stroke. 2009;40:432-8.

69. Rafat N, Beck G, Schulte J, Tuettenberg J, Vajkoczy P. Circulating endothelial progenitor cells in malignant gliomas. J Neurosurg. 2010;112:43-9.

70. Nowak K, Rafat N, Belle S, et al. Circulating endothelial progenitor cells are increased in human lung cancer and correlate with stage of disease. Eur J Cardiothorac Surg. 2010;37:758-63.

71. Voswinckel R, Ziegelhoeffer T, Heil M, et al. Circulating vascular progenitor cells do not contribute to compensatory lung growth. Circ Res. 2003;93:372-9.
72. Peichev M, Naiyer AJ, Pereira D, et al. Expression of VEGFR-2 and AC133 by circulating human CD34(+) cells identifies a population of functional endothelial precursors. Blood. 2000;95:952-8.

73. Estes ML, Mund JA, Ingram DA, Case J. Identification of endothelial cells and progenitor cell subsets in human peripheral blood. Curr Protoc Cytom. 2010; Chapter 9:Unit 933 1-11.

74. Medina RJ, O'Neill CL, Sweeney M, et al. Molecular analysis of endothelial progenitor cell (EPC) subtypes reveals two distinct cell populations with different identities. BMC Med Genomics. 2010;3:18.

75. Timmermans F, Plum J, Yoder MC, Ingram DA, Vandekerckhove B, Case J. Endothelial progenitor cells: identity defined? J Cell Mol Med. 2009;13:87-8102.

76. Melero-Martin JM, Khan ZA, Picard A, Wu X, Paruchuri S, Bischoff J. In vivo vasculogenic potential of human bloodderived endothelial progenitor cells. Blood. 2007;109:4761-8.

77. Yamada M, Kubo H, Ishizawa K, Kobayashi S, Shinkawa M, Sasaki H. Increased circulating endothelial progenitor cells in patients with bacterial pneumonia: evidence that bone marrow derived cells contribute to lung repair. Thorax. 2005;60: 410-3.

78. Lam CF, Roan JN, Lee CH, et al. Transplantation of endothelial progenitor cells improves pulmonary endothelial function and gas exchange in rabbits with endotoxin-induced acute lung injury. Anesth Analg. 2011;112:620-7.

79. Aicher A, Zeiher AM, Dimmeler S. Mobilizing endothelial progenitor cells. Hypertension. 2005;45:321-5.

80. Asahara T, Takahashi T, Masuda H, et al. VEGF contributes to postnatal neovascularization by mobilizing bone marrow-derived endothelial progenitor cells. EMBO J. 1999;18:3964-72.

81. Cesari F, Sofi F, Caporale R, et al. Relationship between exercise capacity, endothelial progenitor cells and cytochemokines in patients undergoing cardiac rehabilitation. Thromb Haemost. 2009;101:521-6.

82. Redondo S, Hristov M, Gordillo-Moscoso AA, Ruiz E, Weber C, Tejerina T. High-reproducible flow cytometric endothelial progenitor cell determination in human peripheral blood as CD34+/ CD144+/CD3 - lymphocyte sub-population. J Immunol Methods. 2008;. doi:10.1016/j.jim.2008.02.011.

83. Laufs U, Urhausen A, Werner $\mathrm{N}$, et al. Running exercise of different duration and intensity: effect on endothelial progenitor cells in healthy subjects. Eur J Cardiovasc Prev Rehabil. 2005; 12:407-14.

84. Sandri M, Adams V, Gielen S, et al. Effects of exercise and ischemia on mobilization and functional activation of bloodderived progenitor cells in patients with ischemic syndromes: results of 3 randomized studies. Circulation. 2005;111:3391-9.

85. Paul JD, Powell TM, Thompson M, et al. Endothelial progenitor cell mobilization and increased intravascular nitric oxide in patients undergoing cardiac rehabilitation. J Cardiopulm Rehabil Prev. 2007;27:65-73.

86. Moyna NM, Thompson PD. The effect of physical activity on endothelial function in man. Acta Physiol Scand. 2004;180:113-23.

87. Condon ET, Wang JH, Redmond HP. Surgical injury induces the mobilization of endothelial progenitor cells. Surgery. 2004;135: 657-61.

88. Becchi C, Pillozzi S, Fabbri LP, et al. The increase of endothelial progenitor cells in the peripheral blood: a new parameter for detecting onset and severity of sepsis. Int $\mathrm{J}$ Immunopathol Pharmacol. 2008;21:697-705.

89. Jeong JO, Kim MO, Kim H, et al. Dual angiogenic and neurotrophic effects of bone marrow-derived endothelial progenitor cells on diabetic neuropathy. Circulation. 2009;119:699-708.

90. Rehman J, Li J, Parvathaneni L, et al. Exercise acutely increases circulating endothelial progenitor cells and monocyte-/macrophagederived angiogenic cells. J Am Coll Cardiol. 2004;43:2314-8. 